\title{
Diversification et fragilité des dispositifs de services de conseil agricole au Nord-Cameroun
}

Diversification and fragile devices agricultural advisory services in Northern

Cameroon

Mana Bourou, Michel Havard et Patrice Djamen Nana

\section{OpenEdition}

Journals

Édition électronique

URL : http://journals.openedition.org/economierurale/4132

DOI : 10.4000/economierurale.4132

ISSN : 2105-2581

Éditeur

Société Française d'Économie Rurale (SFER)

Édition imprimée

Date de publication : 15 octobre 2013

Pagination : 91-101

ISSN : 0013-0559

Référence électronique

Mana Bourou, Michel Havard et Patrice Djamen Nana, « Diversification et fragilité des dispositifs de services de conseil agricole au Nord-Cameroun », Économie rurale [En ligne], 337 | septembre-octobre 2013, mis en ligne le 15 octobre 2015, consulté le 19 avril 2019. URL : http://journals.openedition.org/ economierurale/4132 ; DOI : 10.4000/economierurale.4132 


\title{
Diversification et fragilité des dispositifs de services de conseil agricole au Nord-Cameroun
}

\author{
Mana BOUROU • Ministère de l'Agriculture et du Développement Rural, Yaoundé, Cameroun, \\ Université de Liège-Gembloux Agro-Bio Tech, Louvain-la-Neuve, Belgique \\ manabourou@yahoo.fr \\ Michel HAVARD • Cirad, UMR innovation, Montpellier \\ michel.havard@cirad.fr
} Patrice DJAMEN NANA • African Conservation Tillage Network (ACT), Ouagadougou, Burkina Faso patrice.djamen@act-africa.org

Cet article ${ }^{1}$ analyse l'évolution des Dispositifs de services de conseil agricole (DSCA) au NordCameroun, à partir d'enquêtes réalisées sur six des quinze dispositifs identifiés. Les changements socio-économiques marqués par l'émergence des acteurs non étatiques ont accéléré la diversification des DSCA. La pluralité de ces acteurs permet potentiellement de suppléer les difficultés des dispositifs publics et de faciliter l'accès des producteurs aux services agricoles. Mais ces nouveaux dispositifs restent fragiles. La pérennisation, la sécurisation du financement des dispositifs et leur adaptation aux besoins des agriculteurs sont discutées, ainsi que la mobilisation d'un plus grand nombre d'acteurs (État, bailleurs, OP, privés, agriculteurs, recherche et formation agricoles) dans leur fonctionnement.

MOTS-CLÉS : service, dispositif, approche, conseil, pérennisation

\begin{abstract}
Diversification and fragile devices agricultural advisory services in Northern Cameroon This article analyzes the evolution of agricultural advisory services (AAS) in northern Cameroon. Surveys on six of the fifteen AAS identified were carried out. Socio-economic transformations marked by the emergence of non-state actors have accelerated the diversification of AAS. The plurality of AAS can potentially compensate difficulties of public AAS and facilitate producers' access to agricultural services. But these new systems of AAS remain fragile. Their sustainability of these systems, the security of their funding and their adaptation to farmers' needs are discussed, as well as the consequences of the involvement of more actors (government, donors, OP, private farmers, agricultural research and training) in their functioning. (JEL: L3, Q13).
\end{abstract}

KEYWORDS: service, device, approach, advice, sustainability

$\mathbf{E}^{\mathrm{n}}$ Afrique subsaharienne, le secteur agricole est marqué depuis deux décennies par des changements importants accélérés par les Plans d'ajustement structurel agricole (PASA) : le désengagement de l'État

1. Cet article est tiré d'une communication présentée au colloque SFER, Conseil en agriculture : acteurs, marchés, mutations, 14 et 15 octobre 2010, AgroSup Dijon, France. http://www.sfer.asso.fr/ content/download/3619/32627/file/1a-Bourou.pdf. de certains services agricoles, la libéralisation de l'économie et la décentralisation (Heemskerk et al., 2008). Ces changements ont favorisé la diversification des dispositifs de services agricoles (organismes à but non lucratif, organisations agricoles et privés), et ont accru le rôle des bénéficiaires dans ces dispositifs (Moumouni et Labarthe, 2012). Dans le cas du Cameroun, jusqu'au milieu des années 1980, les services aux agriculteurs (formation, 
vulgarisation, fourniture et financement d'intrants et d'équipements agricoles, commercialisation de spéculations comme le coton, le café, le cacao) étaient assurés essentiellement par des organismes publics. Au début des années 1990, l’État camerounais a pris des mesures (exemple de la loi de 1992 sur l'organisation des producteurs) pour favoriser la pluralité des dispositifs de services, et à terme leur pérennité, mais aussi pour responsabiliser davantage leurs bénéficiaires dans la fourniture, le financement et l'accès aux biens et services qui n'étaient plus assurés par l'État. Ces changements de contexte ont aussi mis en évidence les limites de l'approche descendante de la vulgarisation agricole basée sur la diffusion de messages techniques, à l'exemple de l'approche Formations \& Visites (Davis, 2008 ; Wallace, 1997 ; Birner et al., 2009). Cette approche n'est pas opérante pour relever le défi actuel de l'amélioration des capacités de prise de décision des producteurs afin d'assurer la durabilité de leurs exploitations (Djamen et al., 2011). En réaction, des approches, notamment dans le cas du conseil agricole, orientées vers la responsabilisation des agriculteurs (Anandajayasekeram et al., 2008) et leur participation financière au coût du service (Moumouni et al., 2009), ont été développées par la recherche en relation avec des acteurs du développement. Dans les années 2000, certaines recherches visaient à développer des services de conseil agricole répondant aux besoins du marché (Chipeta, 2006), et d'autres à développer le conseil à l'exploitation familiale, s'appuyant sur une approche globale de l'exploitation agricole (Faure et al., 2004 ; Djamen et al., 2003).

Ces changements ont été guidés par l'idée que des Dispositifs de services de conseil agricole (DSCA) pluralistes et une contribution plus importante des agriculteurs garantiraient une plus grande durabilité de ces dispositifs. Cet article vise à mettre à l'épreuve cette idée dans le cas du Cameroun, pays exemplaire des transformations du conseil agricole en Afrique.

\section{Garantir la durabilité des dispositifs de services de conseil}

Les services de conseil rural peuvent être définis comme les différentes activités donnant les informations et les services voulus et demandés par les acteurs du monde rural pour développer eux-mêmes leurs propres capacités techniques, organisationnelles et managériales dans le but d'améliorer leurs conditions de vie (Adolph, 2011). Le conseil est un service immatériel qui permet de formuler des questions, d'identifier des solutions, faciliter la mise en œuvre d'actions et évaluer les résultats (Faure, 2007). Le conseil agricole aide le producteur à atteindre ses objectifs en lui apportant un regard extérieur sur sa situation et ses possibilités (Chombart de Lauwe et al., 1969). Les services sont fournis au travers d'un dispositif qui peut être défini comme l'ensemble des moyens mobilisés pour atteindre un objectif précis. Les relations entre les services peuvent s'analyser en termes de compétition, de coordination ou de coopération et posent des questions, notamment sur les mécanismes de financement et de gouvernance, d'autonomie et d'indicateurs de leur durabilité (Faure, 2007).

Les DSCA peuvent être analysés à l'aide de différents cadres. Celui de Birner et al. (2009) est à la base de la grille élaborée pour l'analyse de la structure et du fonctionnement des dispositifs de conseil de notre échantillon. Cette grille prend en compte les éléments suivants : les structures de gouvernance (État, privés, agriculteurs), les capacités (ressources humaines, infrastructures, ressources financières), les modes de gestion (approche, rôles, résultats, évaluation), et les méthodes du conseil (types de formation, de transfert, individuel, groupe, masse, contenu). Les éléments de contexte 
(histoire, compétences des prestataires, types d'activités agricoles, etc.) qui influent sur la structuration et l'organisation des services sont également considérés.

Il s'agit ici de comprendre si certaines transformations des DSCA (leur pluralité, une contribution plus importante des bénéficiaires) favorisent ou non la durabilité de ces dispositifs. Pour analyser cette durabilité, nous mettons l'accent sur la gouvernance par différents types d'acteurs, sur la sécurisation du financement (car les bénéficiaires y contribuaient très peu jusqu'à présent), et sur les ressources humaines, c'està-dire les agents des dispositifs qui doivent s'approprier de nouvelles approches et façons de travailler. Nous nous focalisons aussi sur la contribution et la participation des agriculteurs aux DSCA, souvent perçue comme un facteur de succès et de pérennisation des dispositifs (Mercoiret, 1994).

La grille d'analyse sur les institutions des Systèmes nationaux de conseil agricole (SNCA) (Labarthe et Laurent, 2011) a été mobilisée pour discuter des compétences et des besoins des agents des DSCA, de leurs relations avec les agriculteurs, mais aussi de la motivation des agriculteurs, et de la capitalisation des connaissances. L'étude sur l'analyse des incitations techniques, financières et organisationnelles pour motiver les agriculteurs à financer la recherche et la vulgarisation agricoles (Moumouni et al., 2009) a été mobilisée sur la question de la participation des agriculteurs aux DSCA. Ces incitations renvoient ici à une stratégie de participation croissante des paysans et de leurs organisations au financement des services, de prise en compte des besoins des paysans, et de valorisation des leaders agricoles locaux.

\section{Méthodologie}

Cette étude a été réalisée au Nord-Cameroun entre 2007 et 2008 par l'Institut de recherche agricole pour le développement (IRAD).

\section{Présentation de l'étude de cas sur les DSCA}

Les DSCA publics sont issus des ministères de l'Agriculture et du Développement rural (Minader), de l'Élevage, des Pêches et des Industries Animales (Minépia), et de la Société de développement du coton au Cameroun (Sodécoton). Ces dispositifs évoluent pour prendre en compte les changements de l'environnement institutionnel et socio-économique. Il est notamment question de promouvoir les organisations agricoles (à l'exemple de la Confédération nationale des producteurs de coton du Cameroun [CNPCC] ) et le conseil agricole au travers de l'approche de Conseil à l'exploitation familiale agricole (CEF) (Djamen et al., 2003) dont l'appropriation exige des ajustements technique, organisationnel, économique et social (Djamen et al., 2011). Le Minader et le Minépia mettent aussi l'accent sur la formation pour une meilleure insertion professionnelle des jeunes, par la rénovation du dispositif d'enseignement agricole. Le processus de désengagement de l'État a favorisé la recomposition des DSCA au Nord-Cameroun. Cette recomposition se traduit par de nouveaux rôles et activités dans les structures existantes, par le développement de nouveaux organismes à but non lucratif, et des organisations agricoles assurant des services de conseil agricole. Elle permet de diversifier les offres de services et donc de prendre en compte différents bénéficiaires et demandes.

Les organismes publics ou parapublics vulgarisent des thèmes techniques selon une approche « descendante » qui évolue lentement du « directif » au «participatif ». Le dialogue avec le paysan prend de l'importance, les Organisations de producteurs (OP) et Groupements de producteurs (GP) jouent de plus en plus un rôle d'interface entre ces organismes publics et les paysans. Mais les compétences des 
vulgarisateurs ne sont pas en adéquation avec ces changements d'approche, les financements sont également insuffisants. Ce qui amène ces organismes à mettre en œuvre des réformes. Ainsi, la Sodécoton donne la priorité aux GP qui ont un bon fonctionnement interne et de bons résultats technico-économiques. Par ailleurs, la Sodécoton conduit des actions de renforcement des capacités du personnel d'encadrement par la formation et la mise en œuvre du conseil, etc.

Les organismes à but non lucratif $(\mathrm{ONG}$, associations confessionnelles, etc.) interviennent dans la production de biens et de services logistiques, informationnels, relationnels et professionnels destinés en priorité aux groupes vulnérables (pauvres, femmes, jeunes) en milieu rural, et pas seulement agricole. Ces services sont financés par des bailleurs de fonds (fondations, associations internationales) et par une contribution limitée des bénéficiaires. Les approches développées visent la promotion, la structuration et la responsabilisation des groupes d'agriculteurs. Nombreux sont ces dispositifs qui ont été confrontés à des dysfonctionnements dans la gestion des groupes bénéficiaires, à un faible niveau des animateurs relais et à des difficultés de financement.

L'émergence des organisations agricoles (GP, OP) et des faitières (unions, fédérations), a été favorisée par l'adoption de la loi sur les Groupes d'initiative commune (GIC) en 1992. Ces organisations agricoles ont été créées principalement autour des filières grâce aux appuis technique et financier de partenaires nationaux et internationaux. Les approches développées visent à responsabiliser les paysans dans la gestion de ces organisations et à atteindre l'autonomie financière, autour d'activités diversifiées. Ces organisations rencontrent des difficultés pour recouvrer les cotisations des producteurs et dans la gestion de leurs activités.
Ces descriptions du rôle, du fonctionnement et des activités des différents types de DSCA, font ressortir des questions communes à ces dispositifs, i) sur leur pérennité, et plus spécifiquement leur financement, mais aussi ii) sur la nécessité d'adapter les compétences et les profils des agents des DSCA aux nouvelles approches développées, et enfin, iii) sur les difficultés rencontrées pour faire participer les agriculteurs au fonctionnement des dispositifs.

\section{Dispositif de recueil et d'analyse des données}

L'étude a été réalisée en deux étapes.

- En premier lieu, des contacts avec des personnes-ressources et une étude bibliographique ont permis d'inventorier quinze DSCA, et de choisir un échantillon de dispositifs à étudier. Les DSCA ont été classés en trois groupes caractérisant leur pluralité : (i) les organismes publics (5), (ii) les organismes à but non lucratifs (5) et (iii) les coopératives et organisations de producteurs (5). Pour tester l'hypothèse que la pluralité pouvait accroître la pérennité des DSCA, deux dispositifs ont été retenus dans chaque groupe. Le choix des dispositifs a été guidé par l'existence d'informations et par la disponibilité des principaux acteurs à fournir des informations, et à répondre aux entretiens (tableau 1) : organismes publics - Programme national de vulgarisation et de recherche agricole (PNVRA), Sodécoton - ; organismes à but non lucratif (ONG) - Service d'appui aux initiatives locales de développement (Saild), Centre d'éducation rurale de Ngong (CERN) - ; coopératives et Organisations des producteurs (OP) - Association des producteurs et stockeurs de céréales du Diamaré (Aprostoc), Coopérative de producteurs d'oignon de la province du Nord-Cameroun (Tignéré). 
RECHERCHE

Mana BOUROU, Michel HAVARD, Patrice DJAMEN NANA

Tableau 1. Présentation des dispositifs analysés

\begin{tabular}{|c|c|c|c|c|c|c|c|}
\hline \multirow{2}{*}{$\begin{array}{l}\text { Dispositifs } \\
\text { Structures }\end{array}$} & & \multicolumn{2}{|c|}{ Organismes à but non lucratifs } & \multicolumn{2}{|c|}{ Organismes publics } & \multicolumn{2}{|c|}{ Organisations de producteurs } \\
\hline & & SAILD & CERN & SODECOTON & PNVRA & APROSTOC & TIGNERE \\
\hline \multirow[t]{2}{*}{$\begin{array}{l}\text { Responsables } \\
\text { dispositifs }\end{array}$} & effectif & $\begin{array}{c}1 \text { chef } \\
\text { d'antenne } \\
4 \text { chefs de } \\
\text { programme }\end{array}$ & $\begin{array}{l}1 \text { coordinateur } \\
9 \text { Animateurs }\end{array}$ & $\begin{array}{c}9 \text { CDR } \\
110 \text { CDS }\end{array}$ & $\begin{array}{l}1 S R \\
2 \mathrm{TS} \\
4 \mathrm{SS}\end{array}$ & $\begin{array}{l}9 \text { Responsables } \\
\text { paysans }\end{array}$ & $\begin{array}{l}\text { bureau } \\
\text { exécutif }\end{array}$ \\
\hline & \% enquêtés & $100 \%$ & $100 \%$ & $10 \%$ & $100 \%$ & $100 \%$ & $100 \%$ \\
\hline \multirow{2}{*}{$\begin{array}{l}\text { Conseillers } \\
\text { (AR, CP, AVZ, } \\
\text { CDZ) }\end{array}$} & Effectif & 50 & 48 & 250 & 46 & 12 & $\begin{array}{c}\text { Service } \\
\text { technique * }\end{array}$ \\
\hline & $\%$ enquêtés & $10 \%$ & $10 \%$ & $5 \%$ & $10 \%$ & $50 \%$ & $100 \%$ \\
\hline \multirow{2}{*}{$\begin{array}{l}\text { Bénéficiaires } \\
\text { (Groupes) }\end{array}$} & GIC/ OP/GP & 450 & 48 & 1900 & 425 & $\begin{array}{c}220 \text { stockeurs } \\
40 \text { producteurs }\end{array}$ & 8 \\
\hline & $\begin{array}{c}\text { Unions } \\
\text {-fédérations }\end{array}$ & 49 & 6 & 1 & ND & 9 & 5 \\
\hline \multirow{2}{*}{ EFA } & Effectif & 6000 & 500 & 370000 & ND & 52000 & 1200 \\
\hline & $\%$ femmes & $59 \%$ & $40 \%$ & ND & ND & ND & ND \\
\hline \multicolumn{2}{|c|}{ Activités principales } & $\begin{array}{c}\text { Sécurité } \\
\text { alimentaire }\end{array}$ & Formation & Coton & Vulgarisation & Sécurité alimentaire & $\begin{array}{c}\text { Profession- } \\
\text { nalisation }\end{array}$ \\
\hline \multicolumn{2}{|l|}{ Adhésion } & Libre & Libre & Contrat & Libre, Contrat & Libre & Libre \\
\hline
\end{tabular}

Notes : AR. Animateur relais, AVZ. Agent vulgarisateur de zone, CDR. Chef de région, CDS. Chef de secteur, CDZ. Chef de zone, CP. Conseiller paysan, EFA. Exploitation familiale agricole, GIC. Groupe d'initiative commune, OP. Organisation de Producteurs, GP. Groupement de producteurs, SR. Superviseur régional, SS. Superviseur secteur, TS. Technicien spécialisé. * Prestataire privé

Source : les auteurs.

- En second lieu, la grille élaborée pour l'analyse de la structure et du fonctionnement des DSCA a servi de fil conducteur à la réalisation des entretiens et au choix de l'échantillon des personnes à interviewer :

- une quarantaine de responsables de dispositifs ont été interviewés sur l'historique, les zones d'intervention, les activités, les services, le public ciblé, les difficultés et les perspectives de leur dispositif ; ces éléments ont servi à l'élaboration des monographies de chaque dispositif (tableau 2), et aux discussions sur la pérennité de la pluralité des dispositifs, et la participation des agriculteurs ;

- une quarantaine d'agents (conseillers, animateurs) chargés de la mise en œuvre des activités de terrain ont été interviewés sur leur statut, les thèmes développés, les cibles de leurs interventions et leurs difficultés afin d'appréhender l'adéquation de leurs profils et compétences aux évolutions des approches de conseil.

\section{La pérennité des dispositifs de services de conseil agricole en question}

Les monographies et analyses comparatives des six dispositifs étudiés questionnent la pérennité des DSCA comme dans d'autres situations d'Afrique subsaharienne (Spinat et al., 2006 ; Havard et al., 2007). Cette pérennité des dispositifs est analysée, au travers de la sécurisation de leurs financements, des compétences de leurs personnels, de l'accès et de la participation des agriculteurs bénéficiaires aux services.

\section{Sécuriser les financements des DSCA}

Tous les DSCA étudiés ont réussi à mettre en place des GP, et certains des fédérations, qui gagnent progressivement en autonomie. Mais pratiquement tous rencontrent des difficultés liées au financement des services non rémunérateurs comme le conseil et la formation. Seule la Sodécoton 


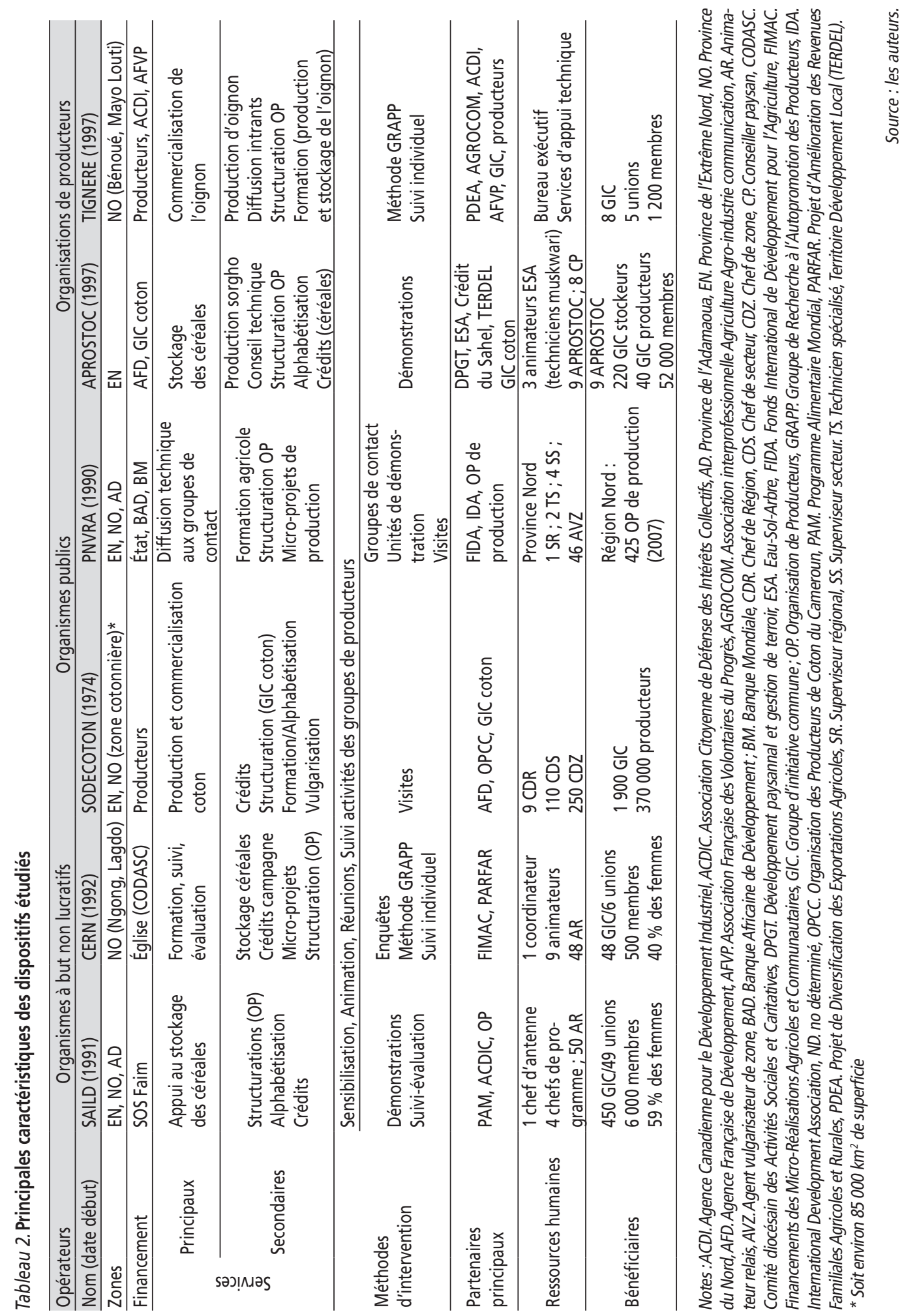

96 • Économie Rurale 337/Septembre-Octobre 2013 
continue à financer et assurer ses services par les prélèvements directs sur la vente du coton. Mais, dans un souci de durabilité et de rentabilité de son dispositif, elle cible davantage les GP qui peuvent effectivement contribuer au fonctionnement de son dispositif, grâce à de bonnes performances technico-économiques (niveau de production, taux de remboursement des crédits). Les producteurs démunis, ceux n'ayant pas accès à des filières marchandes, et ceux non solvables pour la Sodécoton, ne peuvent plus avoir accès aux services.

Dans les organismes à but non lucratif (Cern, Saild) et les OP (Aprostoc, Tignéré), la gestion simultanée de services rémunérateurs (approvisionnement en intrants) et à caractère social plus ou moins subventionnés (lutte contre la pauvreté, appui à des groupes défavorisés et à des agriculteurs en difficultés) s'avère très délicate en termes de financement et de relations des responsables avec les producteurs. Ces dispositifs bénéficient d'appuis technique et financier de partenaires nationaux et internationaux, alors que les contributions (cotisations, adhésions, prélèvements etc.) des bénéficiaires sont insuffisantes et variables selon les opérateurs. L'irrégularité des financements extérieurs ne permet pas d'assurer la continuité des services, ce qui provoque des tensions au sein des dispositifs, et avec les bénéficiaires (Cern et Saild). Quand les dispositifs sont défaillants, la confiance entre les acteurs impliqués est altérée, ce qui est une contrainte forte à la contribution des bénéficiaires au financement des services de conseil agricole, comme signalé dans d'autres études (Moumouni et al., 2009).

Les agriculteurs s'engagent souvent plus pour leurs intérêts individuels que pour des objectifs communs comme l'appartenance et le partage des valeurs d'un groupe dont les résultats des activités bénéficient à l'ensemble de la communauté (Martinot, 2007). Il en résulte aussi que de nombreux producteurs font alors partie d'un GP pour bénéficier des appuis, y compris les crédits (intrants, argent), avec l'intention inavouée de ne rien avoir à rembourser. Cette motivation opportuniste liée à l'inadaptation des incitations financières n'est pas durable (Moumouni et al., 2009).

Quand les contributions en espèces sont difficiles à mobiliser, diverses formes de contributions sont possibles (travail, matériaux locaux, temps, etc.), mais l'efficacité de la participation des agriculteurs à long terme et leur responsabilisation sont alors discutables (Moumouni et al., 2009).

La sécurisation des financements des DSCA ne peut alors être assurée par l'autofinancement que pour des services rémunérateurs (approvisionnement en intrants, commercialisation des produits) et des bénéficiaires solvables. La sécurisation des financements nécessite de recourir à des subventions partielles ou totales pour la mise en œuvre de services non rémunérateurs (conseil, formation), et pour toucher des bénéficiaires appartenant à des groupes vulnérables (agriculteurs démunis, femmes, jeunes, etc.).

Ces difficultés mettent en cause la pérennisation de ces dispositifs qui sont alors contraints de se restructurer, de revoir leurs stratégies pour s'adapter aux changements de leur environnement et pour ne pas disparaître.

\section{Développer les compétences des agents des DSCA}

Le conseil agricole est basé sur la relation entre un prestataire et un bénéficiaire. Il est donc important qu'un conseiller soit en mesure de bien appréhender sa relation avec le bénéficiaire (OP, agriculteurs) afin d'identifier précisément les besoins, les connaissances et la motivation des bénéficiaires pour un service donné (Labarthe et Laurent, 2011). Le conseiller doit être en mesure d'aider le bénéficiaire à bien 
identifier le problème et à produire les solutions appropriées. Le degré d'interaction entre le conseiller et le bénéficiaire varie, il est plus élevé dans le conseil individuel que dans le conseil de groupe.

Mais au Nord-Cameroun, le fort taux d'analphabétisme des agriculteurs et les faibles compétences des cadres chargés de l'animation des dispositifs sont des contraintes fortes dans la relation entre le conseiller et l'agriculteur, et dans la gestion des services rémunérateurs. En effet, les faibles compétences de leurs responsables ont amené certains DSCA à abandonner des services rémunérateurs (Aprostoc, Tignéré), et ont provoqué des tensions et une perte de confiance entre les agriculteurs et les responsables (coopérative de Tignéré). Il est donc important que l'État soutienne davantage l'enseignement primaire et agricole et, en partenariat avec les OP, la formation continue des producteurs (alphabétisation, formation professionnelle). Cet appui à la formation permettrait aux producteurs de participer plus efficacement à la gestion des GP et à la mise en œuvre des services d'appui-conseil.

Les questions de compétence, de statut et d'identité des conseillers et des responsables de dispositifs d'appui-conseil sont aussi posées, car c'est sur eux que repose la qualité du travail réalisé (Djamen et al., 2011). Les expériences de formation des conseillers sont nettement insuffisantes. Elles sont réalisées par des prestataires locaux ou étrangers dans le cas du programme d'appui à la compétitivité des exploitations familiales agricole (Acefa). Les cadres (ingénieurs) chargés du fonctionnement des dispositifs n'ont pas, pour la plupart, de compétences en animation et n'ont pas été formés aux approches et à la gouvernance de ces dispositifs. Former ces conseillers agricoles et ces responsables demande de revoir les parcours de formation initiale en agriculture vers l'acquisition de compétences. Pour que la relation conseiller-agriculteur fonctionne bien, soit efficace, le conseiller doit pouvoir accéder à des connaissances, des références en rapport avec le problème identifié avec l'agriculteur et adaptées aux caractéristiques des exploitations. Les références du conseiller doivent être combinées avec les savoirs des agriculteurs pour être pertinentes. Il est donc nécessaire de disposer de lieux pour capitaliser les connaissances, élaborer des référentiels, assurer une veille technologique, etc. (Labarthe et Laurent, 2011).

\section{Favoriser l'accès et la participation des agriculteurs aux services de conseil agricole}

Selon Mercoiret (1994), les expériences passées d'appui aux producteurs ont montré que les chances de succès et de pérennisation des interventions étaient meilleures quand les paysans étaient associés à la gestion des dispositifs. Même si cette participation est parfois une caution ou un alibi, elle vise à garantir une réelle adéquation des services aux besoins des paysans, assurer une insertion des activités dans les réseaux socioprofessionnels, mobiliser les leaders d'opinion, légitimer les organisations paysannes comme interlocuteurs fiables des services étatiques (Faure, 2007). Ceci montre que les questions d'accès et de participation aux services de conseil agricole ne se résument pas à des conditions de coût, mais s'inscrivent dans des rapports sociaux (Labarthe et Laurent, 2011) où la motivation des bénéficiaires pour les services qui leur sont proposés est primordiale (Moumouni et al. 2009). Djamen et al. (2003) avaient noté que certains producteurs du Nord-Cameroun n'étaient pas intéressés par la démarche de conseil à l'exploitation parce qu'ils estimaient ne pas produire suffisamment pour avoir à gérer et en outre ne savaient pas lire et écrire.

La participation des producteurs aux coûts des services est une nécessité, mais 
les questions du niveau de contribution et du délai pour l'atteindre restent posées (Spinat et al., 2006). Ceci est particulièrement vrai quand la majorité des producteurs sont pauvres et que les dispositifs publics de service n'ont plus les moyens pour subventionner leurs interventions comme au Cameroun.

La participation des agriculteurs aux dispositifs de services a été favorisée par les lois de 1990 portant sur la liberté d'association et de 1992 sur la création des sociétés coopératives, et groupes d'initiative commune. Les organismes publics d'appui aux agriculteurs et les ONG ont contribué à la mise en place des groupements, organisations, unions et fédérations d'agriculteurs. Ces organisations sont devenues des bénéficiaires et partenaires (cas des GP coton), mais aussi des prestataires de services euxmêmes (cas de Aprostoc et Tignéré). Cependant, la gestion des services déployés par des OP exige qu'elles soient plus autonomes sur le plan institutionnel, c'està-dire qu'elles possèdent des capacités techniques, financières et managériales. Le programme Acefa, basé sur le conseil agricole est un exemple actuel d'accompagnement de la responsabilisation des producteurs. Son dispositif d'appui conseil est cogéré par l'État et la profession au travers la plate-forme nationale des $\mathrm{OP} d u$ Cameroun (Planopac). Des délégués des groupements de producteurs, des représentants de la cellule technique départementale du Minader et du Minépia sont représentés dans les instances départementales de pilotage du dispositif.

Tous les dispositifs étudiés ont mis en œuvre (ou testés) des démarches visant une participation accrue et centrée sur les projets des bénéficiaires où la co-production de savoir par les conseillers et les agriculteurs est désormais admise : conseil de gestion, écoles paysannes, aide à la conduite des projets de production de l'OP ou de l'exploitant. Pour Faure (2007), le succès des dispositifs de conseil peut être envisageable si ceux-ci sont co-construits par les acteurs impliqués (organisation paysanne, État, ONG...). Mais le fort taux d'analphabétisme des producteurs et le nombre insuffisant de lettrés pour assurer les fonctions des bureaux des GP sont des freins importants à la participation effective des producteurs au pilotage des dispositifs. Il en résulte l'instabilité des GP, ainsi que des difficultés des producteurs à contrôler les activités du bureau de leurs GP, à respecter leurs engagements, à exprimer clairement leurs demandes au conseiller agricole et à participer avec lui à la recherche des solutions.

Les principales incitations à la motivation des agriculteurs pour les services de conseil agricole proposés sont une contribution croissante des agriculteurs et de leurs organisations au fonctionnement des services de conseil agricole, la prise en compte des besoins des agriculteurs par les prestataires de services, et le recours à des leaders agricoles locaux (délégués de GP, chefs de village). Selon Moumouni et al. (2009), la prise en compte des besoins des agriculteurs et le recours à des leaders agricoles peuvent conduire à une motivation durable, alors que la stratégie de participation financière des agriculteurs risque d'échouer s'il n'y pas d'incitations techniques qui réussissent. Mais, dans la réalité, c'est une combinaison de ces stratégies qui est mise en œuvre.

Cette participation des agriculteurs doit aussi permettre de diversifier les formes de soutiens aux demandes variées des agriculteurs, et non pas un soutien uniforme de la demande, comme dans les anciennes politiques de conseil agricole.

Enfin, le système d'information aux producteurs facilite la réalisation des services au sein d'un dispositif. Il permet à court terme aux responsables des dispositifs de réaliser leurs tâches de gestion : diagnostiquer, prévoir, suivre et évaluer. Il favorise aussi des processus d'apprentissage 
individuels et collectifs des conseillers des organisations et représentants de producteurs mais aussi des membres (Faure, 2007).

\section{Conclusion}

$\mathrm{Au}$ Nord-Cameroun, le désengagement de l'État du financement et de la gestion de DSCA au cours des deux dernières décennies a favorisé l'émergence d'organisations diverses (ONG, OP, privés), permettant potentiellement de suppléer les difficultés des dispositifs publics et de faciliter davantage l'accès des producteurs aux services agricoles. Mais ces nouveaux dispositifs sont fragiles. La participation des agriculteurs et de leurs organisations au financement des services est la principale mesure incitative pour amener les agriculteurs à contribuer au fonctionnement des dispositifs. Mais, bien que croissante, cette mesure est encore insuffisante.

La sécurisation des financements n'est assurée que pour les services rémunérateurs et pour les producteurs et organisations de producteurs solvables. Les questions de subventions et de participation des bénéficiaires dans la mise en œuvre de services non rémunérateurs (conseil, formation), et pour toucher des bénéficiaires appartenant à des groupes vulnérables (agriculteurs démunis, femmes, jeunes, etc.) restent posées.

Malgré ces difficultés, la promotion de dispositifs de services agricoles publics et privés pertinents, diversifiés et durables est un atout permettant de prendre en compte les aspirations et demandes de plus de bénéficiaires. Les agriculteurs peuvent alors mieux orienter leurs attentes et s'adresser à l'offre de service qui leur paraît la plus pertinente. Mais la pérennisation et l'adaptation des dispositifs de services agricoles aux besoins des agriculteurs et au contexte socio-économique demeure un véritable défi. Elles demandent en premier lieu une volonté politique avérée pour mettre en œuvre et accompagner les changements institutionnels nécessaires, et mobiliser la contribution d'un grand nombre d'acteurs (État, bailleurs, OP, privés, agriculteurs, recherche et formation agricoles). L'État doit renforcer sa contribution dans l'éducation de base, l'alphabétisation et la formation professionnelle des agriculteurs, ainsi que dans la formation et l'élaboration d'un statut des conseillers agricoles. Les OP en concertation avec l'État ont un rôle important à jouer dans ces dispositifs (conception, mise en œuvre, financement, ressources humaines, etc.) et doivent faire la différence entre leurs fonctions sociales (accompagnement de l'organisation des agriculteurs et défense de leurs intérêts), économiques et techniques (appui-conseil, approvisionnement, commercialisation etc.).

La recherche d'accompagnement est indispensable pour participer à la conception des dispositifs, adapter les démarches d'intervention pour la mise en œuvre des services, et contribuer à la définition d'indicateurs d'évaluation des services au sein des dispositifs.

Les auteurs remercient le Pôle de Recherche Appliquée au Développement des Systèmes Agricoles d'Afrique Centrale (Prasac) qui a rendu possible la réalisation de ce travail. http:// www.prasac-cemac.org/ 


\section{RÉFÉRENCES BIBLIOGRAPHIQUES}

Adolph B. (2011). Rural Advisory Services Worlwide: A Synthesis of Actors and Issues. Global Forum for Rural Advisory Services, Lindau, Switzerland, 42 p. + annexes.

Anandajayasekeram P., Puskur R., Workneh S., Hoekstra D. (2008). Concepts and practices in agricultural extension in developing countries: A source book. IFPRI, Washington, DC, USA, and ILRI, Nairobi, Kenya, 275 p.

Birner R., Davis K., Pender J., Nkonya E., Anandajayasekeram P., Ekboir J., Mbabu A., Spielman D., Horna D., Benin S., Cohen M. (2009). From "Best Pratice" to "Best Fit". A framework for analyzing pluralistic agricultural advisory services worldwide. Journal of Agricultural Education and Extension, vol. $15, n^{\circ} 4$, p. 341-355.

Chipeta S. (2006). Demand Driven Agricultural Advisory Services. Lindau, Suisse, Neuchatel Group, GTZ, SDC, AGRIDEA.

Chombart de Lauwe J., Poitevin J., Tirel J. C. (1969). Nouvelle gestion des exploitations agricoles. $2^{\mathrm{e}}$ édition, Paris, Dunod, 509 p.

Davis K. (2008). Extension in Sub-Saharan Africa: Overview and Assessment of Past and Current Models and Future Prospects. Journal of Agricultural Education and Extension, $\mathrm{n}^{\circ} 15$, p. 15-28.

Djamen Nana P., Djonnewa A., Havard M., Legile A. (2003). Former et conseiller les agriculteurs du Nord-Cameroun pour renforcer leurs capacités de prise de décision. $\mathrm{Ca}$ hiers Agricultures, vol. 12, n 4, p. 241-245.

Djamen Nana P., Havard M., Wey J. (2011). L'évolution du conseil agricole au Nord Cameroun, source d'incertitudes pour les conseillers. $\mathrm{Ca}$ hiers Agricultures, vol. ${ }^{\circ} 20, \mathrm{n}^{\circ}$ 5, p. 370-375. Doi : 10.1684/agr.2011.0506 doi: 10.1684/ agr.2011.0506.

Faure G., Dugué P., Beauval V. (2004). Conseil à l'exploitation familiale : expérience en Afrique de l'ouest et du centre. Édition ministère des Affaires Étrangères, CIRAD, Montpellier, $128 \mathrm{p}$.

Faure G. (2007). L'exploitation agricole dans un environnement changeant : innovation, aide à la décision et processus d'accompagnement.
Habilitation à Diriger des Recherches, Université de Bourgogne, 222 p.

Havard M., Dugué P., Coulibaly Y. (2007). Mali : aider les paysans à mieux gérer leur exploitation. Travaux et Innovations, $\mathrm{n}^{\circ} 138$, p. 46-50.

Heemskerk W., Nederlof S., Wennink B. (2008). Outsourcing agricultural advisory services. Enhancing rural innovation in Sub-Saharan Africa. Amsterdam, Bulletin 380, KIT Publishers, 148 p.

Labarthe P., Laurent C. (2011). Économie des services et politiques publiques de conseil agricole, Cahiers Agricultures, vol. 20, $\mathrm{n}^{\circ} 5$, p. 343-351.

Martinot G. (2007). Trust and contracting in Agri-Food Hyrbid structures. In Fritz M., Rickert U., Chiefer G. (Eds.), Proceeding of the $1^{\text {st }}$ international Forum on Innovation and System Dynamics in Food Networks, February 15-17, Innsbruck-Igls, Austria, p. 483-496.

Mercoiret M. R. (1994). L'appui aux producteurs ruraux. Guide à l'usage des agents de développement et des responsables de groupements. Paris, Khartala, 464 p.

Moumouni I., Vodouhé S. D., Streiffeler F. (2009). What Makes Small-Scale Farmers Participate in Financing Agricultural Research and Extension? Analysis of Three Case Studies from Benin. Journal of Agricultural Extension and Education, vol. 15, n 3, p. 301-316.

Moumouni I., Labarthe P. (2012). Institutionalization of knowledge sharing platforms in the last three decades in Francophone Sub Saharan Africa. 10th European IFSA Symposium, Producing and reproducing farming systems: new modes of organization for the sustainable food systems of tomorrow, Aarhus, Denmark, July 1-4.

Spinat J. B., Traoré B., Saywell A. S. (2006). Appui et conseil aux organisations paysannes en zone Office du Niger. Du projet centre de prestations de services aux «Faranfasi So ». Traverses, $\mathrm{n}^{\circ}$ 16, 41 p.

Wallace I. (1997). Agricultural education at the crossroads: present dilemmas and possible options for the future in Sub-Saharan Africa. International Journal of Educational Development, vol. 17, $\mathrm{n}^{\circ} 1$, p. 27-39. 